\title{
Morbilidad postoperatoria en pacientes con hidatidosis hepática no complicada. Utilización de una propuesta de clasificación de complicaciones*
}

\author{
Drs. CARLOS MANTEROLA D. ${ }^{1}$, Ints. JAVIER MORAGA C. ${ }^{1}$, \\ SEBASTIÁN URRUTIA V. ${ }^{1}$, GRUPO MINCIR \\ 1 Departamento de Cirugía y Traumatología, Facultad de Medicina. Universidad de La Frontera. \\ Temuco, Chile.
}

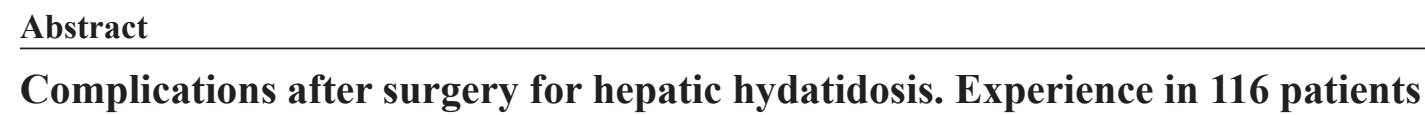

Background: Surgery for hepatic hydatidosis is common in Southern Chile and it is important to know the rates of complications of the procedure. Aim: To report the rates of postoperative complications in patients operated for uncomplicated hepatic hydatidosis. Material and Methods: Prospective study of patients operated for uncomplicated hepatic hydatidosis between 1997 and 2008. The surgical techniques employed were total or partial peri cystectomies, or hepatic resection. Results: One hundred sixteen patients aged $43 \pm$ 17 years, $60 \%$ females, were included in the series. Median cyst diameter was $15 \mathrm{~cm}$. Ten patients $(8.6 \%)$ had complications. Three patients had an atelectasis, two had an acute bronchitis, one had a urinary tract infection, two had surgical wound infections, one developed a residual cavity and one had an eventration. During a median follow up of 95 months, no patient died and one had a recurrence of the disease. Conclusions: Surgery for uncomplicated hydatidosis has a low rate of complications.

Key words: "Echinococcosis" [MeSH], Hydatidosis [Multi], "Echinococcosis, Hepatic" [Mesh], Hepatic Hydatid Cyst, "Morbidity" [Mesh], "Cohort Studies" [Mesh].

\section{Resumen}

Introducción: La cirugía de la hidatidosis hepática (HH) es habitual en hospitales del sur de Chile. El número de publicaciones relacionadas es escasa y los resultados disímiles. Hablar de morbilidad postoperatoria es complejo debido a la diversidad en la gravedad e implicancias para el paciente; basado en ello, Clavien publicó una clasificación basada en la gravedad de la morbilidad postoperatoria. El objetivo de este estudio es reportar la morbilidad postoperatoria en pacientes intervenidos por $\mathrm{HH}$ no complicada aplicando la clasificación de Clavien. Material y Método: Estudio de cohorte prospectiva. Se incluyeron pacientes intervenidos quirúrgicamente por HH no complicada, en el Hospital Hernán Henríquez Aravena de Temuco, entre 1997 y 2008. La variable resultado principal fue desarrollo de morbilidad postoperatoria aplicando la escala de Clavien. Otras variables de interés fueron estancia hospitalaria, mortalidad y recurrencia. Las técnicas quirúrgicas

*Recibido el 12 de Enero de 2010 y aceptado para su publicación el 10 de Marzo de 2010.

Parcialmente financiado por la Dirección de Investigación de la Universidad de La Frontera.

Correspondencia: Dr. Carlos Manterola D.

Manuel Montt 112, oficina 408, Temuco, Chile.

E-mail: cmantero@ufro.cl 
empleadas fueron periquistectomía total o subtotal y resección hepática. Se calcularon porcentajes, medidas de tendencia central y dispersión; intervalos de confianza de 95\% e incidencia. Resultados: 116 pacientes, con mediana de edad de 40,5 años; $60,3 \%$ de género femenino. La mediana del diámetro ultrasonográfico de los quistes fue de 15,0 cm. El tiempo quirúrgico promedio fue de 115,4 $\pm 23,2 \mathrm{~min}$. La incidencia de morbilidad fue de 8,6\%; 90\% de los cuales eran Grado I o II de Clavien. La etiología fue 5,2\% de complicaciones médicas y $3,4 \%$ de complicaciones quirúrgicas. No se registró mortalidad. Con una mediana de seguimiento de 95 meses, se verificó recurrencia en 1 paciente, lo que representa una incidencia de $0,9 \%$. Conclusiones: Se describe la morbilidad postoperatoria de una cohorte de pacientes con $\mathrm{HH}$ no complicada con énfasis en la gravedad de esta. La morbilidad observada es inferior a la publicada y de bajo nivel de gravedad.

Palabras clave: Hidatidosis, quiste hidatídico hepático, morbilidad, estudios de cohorte.

\section{Introducción}

La enfermedad hidatídica se mantiene como una zoonosis endémica, en especial en la IX Región, donde las tasas de prevalencia persisten elevadas, lo que se asocia no sólo con el tratamiento de más de 40 casos nuevos de hidatidosis hepática $(\mathrm{HH})$ en adultos por año ${ }^{1}$, sino que también con el hallazgo de enfermedad hidatídica en otras localizaciones abdominales menos habituales como peritoneo, bazo, páncreas y riñón ${ }^{2}$.

Siendo aún un problema de salud prevalente en algunas áreas geográficas del planeta, la información publicada respecto de cirugía de la $\mathrm{HH}$ no es tan abundante como en otras áreas de la cirugía; por otra parte, persiste la controversia respecto de la morbilidad de cirugía de la $\mathrm{HH}$. El comportamiento de esta variable a lo largo del tiempo no ha cambiado de forma muy relevante, con reportes heterogéneos, independiente del lugar de producción de los datos. En artículos nacionales de la década de los noventa se notificaron cifras de $49,0 \%$ a $68,5 \%{ }^{3-8}$; números similares a los publicados en artículos extranjeros, en los que se informaron cifras de $34,7 \%$ a $62,5 \%{ }^{9-11}$. Después del año 2000 la realidad no parece haber mejorado sustancialmente, pues se publicaron cifras de morbilidad postoperatoria de $21,3 \%$ a $53,8 \%{ }^{12-17}$, situación que podría explicarse por la heterogeneidad de las poblaciones analizadas en los diversos estudios, en los que suelen incluirse pacientes con HH complicada y no complicada.

Por otro lado, hablar de morbilidad postoperatoria es complejo debido a la multiplicidad de variables que influyen en ella; y por la diversidad en la gravedad de las complicaciones y las implicancias de esta variable en el pronóstico de cada paciente.

Basado en lo anteriormente señalado es que Clavien y cols diseñaron, validaron y publicaron en 2004 y 2009 una nueva clasificación basada en la gravedad de la morbilidad postoperatoria, que las clasifica en 5 grados $^{18,19}$.

Con base en los ítems antes descritos, dado que la cirugía de la $\mathrm{HH}$ sigue siendo habitual en los hospitales de nuestro país (en especial en el sur de Chile); que el número de publicaciones referentes al tema son escasas en nuestro medio; y que los resultados reportados, en especial en términos de morbilidad son aún muy disímiles, nos pareció de interés reportar nuestra experiencia en el tratamiento de la HH no complicada o simple; para lo cual nos planteamos como pregunta de investigación ¿cuál es la morbilidad postoperatoria en pacientes intervenidos por HH no complicada aplicando la clasificación de Clavien y cols?

Por ende, el objetivo general de este estudio es reportar la morbilidad postoperatoria en pacientes intervenidos por $\mathrm{HH}$ no complicada aplicando dicha clasificación; y los objetivos específicos son describir las características clínicas y la evolución postoperatoria en términos de recurrencia y mortalidad de una cohorte de pacientes intervenidos por HH no complicada.

\section{Material y Método}

Tipo de estudio: Cohorte prospectiva sin grupo de comparación.

Población: Se incluyeron pacientes intervenidos quirúrgicamente por el primer autor, por $\mathrm{HH}$ simple, en el Servicio de Cirugía del Hospital Hernán Henríquez Aravena de Temuco, en el período comprendido entre abril de 1997 y abril de 2008. Se excluyeron pacientes intervenidos por vía laparoscópica, sujetos con hidatidosis concomitante en otra localización, pacientes intervenidos previamente por $\mathrm{HH}$, individuos con complicaciones evolutivas de la enfermedad (absceso hepático de origen hidatídico ${ }^{20}$, colangiohidatidosis ${ }^{21}$, tránsito hepato-torácico ${ }^{22}$, peritonitis hidatídica, anafilaxia y fistulización del quiste a alguna estructura del tubo digestivo).

Protocolo de estudio: Se confeccionó una hoja de recogida de datos en las que se registraron las características biodemográficas y clínicas de los pacientes, los hallazgos de laboratorio e imágenes, las características de los quistes, los aspectos quirúrgicos relacionados; y la evolución postoperatoria en 
términos de morbilidad postoperatoria, necesidad de reintervenciones, mortalidad y recurrencia.

Variables de interés: La variable resultado principal fue el desarrollo de morbilidad postoperatoria, la que se trabajó aplicando la clasificación propuesta por Clavien y cols ${ }^{18,19}$ (Tablas 1a y $1 b$ ), para lo que se exigió un seguimiento mínimo de 12 meses. Otras variables de interés fueron estancia hospita- laria, necesidad de reintervenciones, mortalidad y recurrencia.

Protocolo de seguimiento: Se realizó control clínico con laboratorio general, determinación de inmunoglobulinas específicas (ELISA-IgG y ELISA-IgE), radiología de tórax y ultrasonografía abdominal en los meses 1, 6 y 12; y de ahí en adelante una vez por año del postoperatorio.

Tabla 1a. Clasificación de Clavien y cols ${ }^{18,19}$

\begin{tabular}{|c|c|}
\hline Grado & Definición \\
\hline I & $\begin{array}{l}\text { Cualquier desviación del curso postoperatorio normal que no requiera tratamiento farmacológico, endoscópi- } \\
\text { co, quirúrgico o de radiología intervencionista. Se permite aplicación de tratamientos farmacológicos como } \\
\text { antieméticos, antipiréticos, analgésicos, soluciones electrolíticas y fisioterapia. Incluye infección del sitio } \\
\text { operatorio superficial tratable en la cama del paciente }\end{array}$ \\
\hline II & $\begin{array}{l}\text { Se requiere de tratamiento farmacológico con fármacos diferentes a los anteriores. Incluye transfusión de } \\
\text { hemoderivados y nutrición parenteral total }\end{array}$ \\
\hline \multirow[t]{3}{*}{ III } & Requiere intervención quirúrgica, endoscópica o radiológica \\
\hline & Sin anestesia general \\
\hline & Con anestesia general \\
\hline & $\begin{array}{l}\text { Complicación que amenaza la vida y que requieren tratamiento en unidades de cuidados intermedios o in- } \\
\text { tensivos }\end{array}$ \\
\hline & Disfunción de orgánica única (incluye utilización de diálisis) \\
\hline & Disfunción de orgánica múltiple \\
\hline $\mathbf{V}$ & Muerte del paciente \\
\hline Sufijo “d” & $\begin{array}{l}\text { Si el paciente sufre una complicación al momento del alta, al grado respectivo se agrega el sufijo "d" (disabi- } \\
\text { lity). Esto, indica la necesidad de seguimiento para una correcta evaluación de la complicación }\end{array}$ \\
\hline
\end{tabular}

Nota: Adaptación del texto original.

Tabla 1b. Ejemplos de los diferentes $\operatorname{grados}^{18,19}$

\begin{tabular}{|c|c|}
\hline Grado & Ejemplos de situaciones clínicas \\
\hline I & $\begin{array}{l}\text { Fibrilación auricular convertida después de corrección de niveles de potasio. Atelectasia pulmonar que re- } \\
\text { quiere fisioterapia. Diarrea no infecciosa. ISO superficial }\end{array}$ \\
\hline II & $\begin{array}{l}\text { Taquiarritmia que requiere tratamiento farmacológico. Neumonía tratada con antibióticos. Diarrea que requiere } \\
\text { antibióticos. ITU que requiere antibióticos }\end{array}$ \\
\hline III a & $\begin{array}{l}\text { Bradiarritmia que requiere marcapasos con anestesia local. Bilioma que requiere drenaje percutáneo. Cierre } \\
\text { de herida dehiscente no infectada con anestesia local }\end{array}$ \\
\hline III b & $\begin{array}{l}\text { Taponamiento cardíaco que requiere ventana pericárdica. Fístula broncopleural que requiere cierre quirúrgico. } \\
\text { Fístula anastomótica que requiere relaparotomía. ISO que conduce a eventración }\end{array}$ \\
\hline IV a & $\begin{array}{l}\text { Falla cardíaca que conduce a síndrome de bajo débito. Falla respiratoria que requiere intubación. ACV. Pan- } \\
\text { creatitis necrotizante. IRA que requiere diálisis }\end{array}$ \\
\hline IV b & $\begin{array}{l}\text { Falla cardíaca que conduce a síndrome de bajo débito en combinación con falla renal. Falla respiratoria que } \\
\text { requiere intubación asociada a falla renal. ACV con falla respiratoria }\end{array}$ \\
\hline
\end{tabular}

Nota: Adaptación del texto original. ISO: Infección del sitio operatorio. ITU: Infección del tracto urinario. ACV: Accidente cerebro vascular. IRA: Insuficiencia renal aguda. 
Tratamiento realizado: No hubo un tratamiento estándar, pues los procedimientos quirúrgicos empleados se aplicaron de acuerdo a los hallazgos intraoperatorios. Según éstos, se realizó periquistectomía total o subtotal y resección hepática (lobectomía derecha e izquierda).

\section{Definiciones:}

1. Definimos como HH no complicada, a la enfermedad hidatídica de localización hepática exclusiva sin complicaciones evolutivas. Independiente del número de quistes, del diámetro de la lesión principal, de la existencia de comunicaciones biliares y de la relación del quiste con estructuras vasculares peri hepáticas.

2. Definimos como recurrencia a la aparición de hidatidosis abdominal en cualquier localización, después de un año de la cirugía.

Plan de análisis: Se realizó un análisis exploratorio de los datos; posteriormente, se utilizó estadística descriptiva con cálculo de porcentajes, medidas de tendencia central y dispersión (promedios y desviación estándar; mediana y valores extremos) e intervalos de confianza de 95\% (IC 95\%). Finalmente, se calculó incidencia o riesgo absoluto para el desarrollo de morbilidad postoperatoria, necesidad de reintervenciones, mortalidad y recurrencia.

\section{Resultados}

En el período estudiado fueron tratados según este esquema 116 pacientes, sin registrarse pérdidas de seguimiento. La mediana de edad fue de 40,5 años (15 a 84 años); y el 60,3\% de los pacientes (70 casos), era de género femenino.

Treinta y cuatro pacientes $(29,3 \%)$ eran portado- res de alguna patología asociada, la que se pesquisó antes de su intervención quirúrgica; de las que destacan la colelitiasis (19 casos, 16,4\%). Tabla 2.

De los estudios de laboratorio realizados, destacan medianas dentro de valores normales (Tabla 3); y en relación con las mediciones cualitativas de inmunoglobulinas específicas, la de ELISA-IgG fue positiva en el $67,4 \%$ de los casos, y la de ELISA-IgE en el $52,2 \%$ de los casos.

Los 116 sujetos en estudio presentaban un total de 171 quistes de diverso tamaño y localización. La mediana del diámetro ultrasonográfico de la totalidad de los quistes fue de $15,0 \mathrm{~cm}(5 \mathrm{a} 30 \mathrm{~cm})$; las lesiones se localizaron preferentemente en el lóbulo derecho $(64,7 \%)$ y eran de carácter hipoecoico en el $62,3 \%$ de los casos (Tabla 4 ).

Tabla 2. Comorbilidad asociada en los pacientes en estudio $(n=116)$

\begin{tabular}{lcc}
\hline Variable & n casos & \% \\
\hline Patología asociada (\%) & & \\
Sin patología & 82 & 70,7 \\
Colelitiasis & 19 & 16,4 \\
LCFA & 5 & 4,3 \\
Cardiopatía & 4 & 3,5 \\
Diabetes mellitus & 3 & 2,6 \\
Cardiopatía + DM & 1 & 0,9 \\
Insuficiencia renal crónica & 1 & 0,9 \\
Embarazo (*) & 1 & 0,9 \\
Trauma abdominal (**) & 1 & 0,9 \\
\hline
\end{tabular}

LCFA: Limitación crónica al flujo aéreo. DM: Diabetes mellitus. (*): Embarazo de 15 semanas. (**): La HH fue un hallazgo intra operatorio pesquisado durante la laparotomía exploradora por trauma abdominal; y fue resuelto en el mismo tiempo quirúrgico.

Tabla 3. Distribución de variables continuas de los pacientes en estudio $(n=116)$

\begin{tabular}{lcccc}
\hline Variable & Mediana & Valores extremos & Media \pm DE & IC 95\% \\
\hline Edad (años) & 40,5 & $15-84$ & $42,8 \pm 16,9$ & $39,7-45,9$ \\
Hematocrito (\%) & 39,0 & $25-54$ & $38,9 \pm 4,2$ & $38,1-39,7$ \\
Leucocitos (x mm ${ }^{3}$ ) & 7800,0 & $3.400-20.600$ & $8017,9 \pm 2601,7$ & $7539,4-8496,3$ \\
Bilirrubina total (mg\%) & 1,0 & $0,8-1,3$ & $1,0 \pm 0,3$ & $0,9-1,2$ \\
Fosfatasas alcalinas (U/l) & 300,0 & $60-345$ & $444,3 \pm 445.5$ & $362,3-526,2$ \\
ASAT (U/l) & 24,5 & $10-407$ & $39,3 \pm 48,6$ & $30,3-48,2$ \\
ALAT (U/l) & 25,0 & $8-436$ & $41,7 \pm 57,3$ & $31,1-52,2$ \\
Diámetro del quiste (cm) & 15,0 & $5-30$ & $14,9 \pm 5,9$ & $13,8-15,9$ \\
Estancia hospitalaria (días) & 4,0 & $2-27$ & $5,1 \pm 3,0$ & $4,6-5,7$ \\
Seguimiento (meses) & 95,0 & $13-132$ & $97,7 \pm 38,7$ & $23,9-100,5$ \\
\hline
\end{tabular}

DE: Desviación estándar. IC 95\%: Intervalo de confianza de 95\%. 
Tabla 4. Distribución de variables categóricas en los pacientes en estudio $(n=116)$

\begin{tabular}{|c|c|c|}
\hline Variable & $\mathrm{n}$ casos & $\%$ \\
\hline \multicolumn{3}{|l|}{$\begin{array}{l}\text { Características ultrasonográficas } \\
\text { del quiste }(*)\end{array}$} \\
\hline Hipoecoico & 73 & 62,9 \\
\hline Heterogéneo & 40 & 34,5 \\
\hline Hiperecogénico & 3 & 2,6 \\
\hline \multicolumn{3}{|l|}{ Localización del quiste $(*)$} \\
\hline Lóbulo derecho & 79 & 68,1 \\
\hline Lóbulo izquierdo & 24 & 20,7 \\
\hline Bilateral & 13 & 11,2 \\
\hline \multicolumn{3}{|l|}{ Número de quistes } \\
\hline Uno & 82 & 70,7 \\
\hline Dos & 25 & 21,6 \\
\hline Tres o más & 9 & 7,7 \\
\hline \multicolumn{3}{|l|}{ Comunicaciones biliares $(*)$} \\
\hline Ninguna & 45 & 38,8 \\
\hline Una & 41 & 35,4 \\
\hline Dos o más & 30 & 25,8 \\
\hline \multicolumn{3}{|l|}{ Cirugía realizada $(*)$} \\
\hline Periquistectomía total y subtotal & 100 & 86,2 \\
\hline Resección hepática & 16 & 13,8 \\
\hline \multicolumn{3}{|l|}{$\begin{array}{l}\text { Procedimientos quirúrgicos } \\
\text { concomitantes }\end{array}$} \\
\hline Ninguno & 28 & 24,1 \\
\hline Colecistectomía & 68 & 58,6 \\
\hline $\begin{array}{l}\text { Colecistectomía y } \\
\text { coledocostomía }\end{array}$ & 18 & 15,5 \\
\hline Coledocostomía & 1 & 0,9 \\
\hline Gastrectomía & 1 & 0,9 \\
\hline
\end{tabular}

(*): Se refiere a la lesión principal en aquellos pacientes que tenían dos o más quistes.

El tiempo quirúrgico promedio fue de $115,4 \pm$ 23,2 min y el procedimiento quirúrgico utilizado con mayor frecuencia fue la periquistectomía $(86,2 \%)$. El $61,2 \%$ de los quistes principales tenían comunicaciones biliares; y el 25,8\% tenían dos o más. Por otra parte, en el $75,9 \%$ de los casos se realizó de forma concomitante algún otro procedimiento quirúrgico (Tabla 4).

La incidencia o riesgo absoluto de morbilidad postoperatoria fue de $8,6 \%$ (10 pacientes del total a lo largo de todo el período de seguimiento). Al aplicar la clasificación de Clavien y cols, se verificó que en el $90 \%$ de los pacientes en los que se registró morbilidad postoperatoria, esta era Grado I o II. La etiología se desagrupó de la siguiente forma: 6 casos $(5,2 \%)$, de complicaciones médicas ( 3 casos de atelectasia basal derecha, 2 casos de bronquitis aguda postoperatoria y 1 caso de infección del tracto urinario); 4 casos $(3,4 \%)$, de complicaciones quirúrgicas (2 casos de infección del sitio operatorio superficial,
Tabla 5. Morbilidad en los pacientes en estudio $(n=116)$

\begin{tabular}{lrr}
\hline Variable & n casos & $\mathbf{\%}$ \\
\hline Clavien & 106 & 91,38 \\
Grado 0 & 5 & 4,31 \\
Grado I & 4 & 3,45 \\
Grado II & 0 & 0,00 \\
Grado III & 1 & 0,86 \\
Grado IV & 0 & 0,00 \\
Grado V & & \\
Morbilidad & 106 & 91,38 \\
No & 5 & 4,31 \\
Respiratorias & 2 & 1,73 \\
ISO & 1 & 0,86 \\
ITU & 1 & 0,86 \\
Cavidad residual & 1 & 0,86 \\
Hernia incisional &
\end{tabular}

ISO: Infección del sitio operatorio. ITU: Infección del tracto urinario.

1 caso de cavidad residual y 1 caso de eventración [estos dos últimos requirieron de una reintervención quirúrgica; lo que representa una incidencia de reintervenciones de 1,7\%]). Tabla 5.

La mediana del tiempo de hospitalización fue de 4 días (2 a 27 días); y con una mediana de seguimiento de 95 meses (13 a 132 meses), se verificó recurrencia en 1 paciente, lo que representa una incidencia de $0,9 \%$. Este caso se diagnosticó en el control alejado, tres años después de la primera intervención, reoperándose al paciente 5 meses después del diagnóstico. No se registró mortalidad en la serie.

\section{Discusión}

Como se comentó anteriormente, las publicaciones relacionadas con la cirugía de la $\mathrm{HH}$ son relativamente escasas y de bajo nivel de evidencia; es así como al buscar en la base de datos MEDLINE, utilizando los términos MeSH "Echinococcosis, Hepatic/surgery"[Mesh], se obtuvieron 1.852 registros; los que al ser depurados a través de la aplicación del operador booleano NOT ("alveolar echinococcosis", "pulmonary echinococcosis", "lung echinococcosis", "pharmacological treatment", "laparoscopic surgery") y algunos límites (artículos publicados desde 1999 a la fecha de la revisión [04/01/2010], en población humana, adulta; y en idiomas español, inglés y francés), arrojó una cantidad de 172 artículos; ahora, cuando a estos le agregamos límites relacionados con el tipo de diseño de los artículos buscando el mejor nivel de evidencia (ensayos clínicos, metaanálisis, ensayos clínicos controlados y con asigna- 
ción aleatoria), la búsqueda sólo generó un total de 9 artículos. Esto quiere decir, que en la última década más del $90 \%$ de los artículos referentes a cirugía de la $\mathrm{HH}$ siguen siendo series de casos; la mayor parte de las cuales contienen población heterogénea y por ende, con resultados cuya validez interna y externa es objetable. Por otra parte, al realizar una búsqueda con el término libre "quiste hidatídico hepático no complicado" y algunos límites (artículos publicados desde 1999 a la fecha de la revisión [04/01/2010], en población humana, adulta; y en idiomas español, inglés y francés), sólo se obtuvieron 11 artículos; pero 6 de ellos no tenían relación con la búsqueda realizada.

No obstante todo lo anteriormente expuesto, uno de los dilemas permanentes y que a pesar del avance tecnológico persiste como un problema es la morbilidad de la cirugía de la HH. Llama la atención el hecho que en los últimos 7 años se sigan reportando cifras de $21,3 \%$ a $53,8 \%{ }^{12-17}$; sobretodo si estas se miran desde la perspectiva de que se trata de una enfermedad benigna. Es posible que estos guarismos que parecen desproporcionados y dispersos se relacionen a la heterogeneidad de las poblaciones estudiadas; pues como ya se señaló, en ellos, se mezclan pacientes con HH complicada y no complicada, intervenidos en centros con diverso nivel de experiencia en este tipo de cirugía.

Por otra parte, nos parece lógico plantear que este estudio se desarrolló aplicando un tipo de diseño que se considera apropiado para la valoración de aspectos pronósticos, como son los estudios de cohorte; en este caso, de carácter prospectivo y sin grupo de comparación. Este tipo de diseño aporta un nivel de evidencia tipo $1 b^{23}$, y hasta donde conocemos es el único referente a este tema, en el que se ha utilizado este tipo de diseño y se ha efectuado la medición de la variable de interés aplicando la escala de Clavien y cols ${ }^{18,19}$.

También parece interesante mencionar que la comorbilidad objetivada (29,3\% de los casos), puede de alguna forma incidir en el desarrollo de morbilidad postoperatoria; entre otras cosas porque en algunos casos, fue el detonante de la necesidad de realizar cirugías concomitantes (sobre el 75\% de los casos).

Otro aspecto de interés tiene que ver con que a pesar de tratarse de HH no complicada, algo más de un cuarto de los pacientes tenían más de dos comunicaciones biliares, hecho que hemos demostrado que se asocia a un incremento de la morbilidad postoperatoria $^{24,25}$.

El tiempo de hospitalización promedio de 4 días contrasta con los datos publicados previamente que fluctúan entre 6,5 y 23 días ${ }^{13,14,26-29}$.

La incidencia de morbilidad postoperatoria obser- vada $(+)$, nos parece adecuada en relación a estudios previos, porque incluso en los últimos 5 años se han publicado al respecto cifras de entre $10,8 \%$ y $24,3 \%{ }^{12,13,15,27,30}$, porque la gravedad de las complicaciones medidas con el instrumento antes mencionado es mayoritariamente baja y sólo del $40 \%$ de los casos fue de resorte quirúrgico con una incidencia de reintervenciones menor a la reportada $\left(14 \%{ }^{31}\right)$; y porque la morbilidad notificada es analizada a lo largo de un extenso período de seguimiento (95 meses), que en general supera a los reportes previos.

Por otra parte, la incidencia de recurrencia $(0,9 \%)$, también contrasta con cifras anteriores que oscilan entre $1,5 \%$ y $7,3 \% 12,14,26,28,32$.

Finalmente, la ausencia de mortalidad de esta cohorte destaca al compararla con distintas series, en las que el reporte de esta variable varía entre $0,3 \%$ y $3,7 \% \%^{12-15,17,26,29,32,33}$.

\section{Referencias}

1. Servicio de Cirugía Hospital Hernán Henríquez Aravena de Temuco. Servicio de Salud Araucanía Sur. Estadísticas quirúrgicas año 2000.

2. Manterola C, Vial M, Losada H, Fonseca F, Bustos L, Muñoz S, et al. Uncommon locations of abdominal hydatid disease. Trop Doct 2003; 33: 179-180.

3. Sapunar J, Rappoport J, Sapunar J, Cumsille F. Quiste hidatídico hepático: características clínicas, factores pronósticos y resultados quirúrgicos. Parasitol al Día 1989; 13: 52-63.

4. Hermosilla P, Cruzat A, Venturelli A. Hidatidosis hepática. Análisis de 81 casos operados. Rev Chil Cir 1991; 43: 47-50.

5. Pinto P. Hidatidosis hepática: estudio de una serie de 534 casos. Rev Chil Cir 1991; 43: 184-187.

6. Apablaza S, Burmeister R, Benavides, C. Tratamiento del quiste hidatídico hepático. Rev Chil Cir 1992; 44: 424-427.

7. Vallejos C. Postbeca en región de hidatidosis endémica. Rev Chil Cir 1994; 46: 414-417.

8. Camacho J, Reyes J, Pérez A. Cirugía del quiste hidatídico hepático. Rev Chil Cir 1996; 48: 479-482.

9. González D, Vega A, Monti J, Torres M. Enfermedad hidatídica hepática: experiencia de los últimos 13 años en Florida. Cir Urug 1999; 69: 200-203.

10. Sarotto L, Nallar M, Ferraro A, Danguise E, Merello J, Ferraina P. Tratamiento quirúrgico de la hidatidosis hepática: experiencia en el Hospital de Clínicas en los últimos 15 años. Rev Argent Cir 1999; 76: 94-105.

11. Correa S, Culqui C, Pinto M. Hidatidosis hepática: revisión de casos intervenidos quirúrgicamente en el Hospital Militar Central Lima 1990-1999. Rev Gastroenterol Perú 2000; 20: 261-269.

12. Gourgiotis S, Stratopoulos C, Moustafellos P, Dimopoulos N, Papaxoinis G, Vougas V, et al. Surgical 
techniques and treatment for hepatic hydatid cysts. Surg Today 2007; 37: 389-395.

13. Atmatzidis KS, Pavlidis TE, Papaziogas BT, Mirelis C, Papaziogas TB. Recurrence and long-term outcome after open cystectomy with omentoplasty for hepatic hydatid disease in an endemic area. Acta Chir Belg 2005; 105: 198-202.

14. Nari G, Ponce O, Cirami M, Jozami J, Toblli J, Eduardo $\mathrm{M}$, et al. Five years experience in surgical treatment of liver hydatidosis. Int Surg 2003; 88: 194-198.

15. Agayev RM, Agayev BA. Hepatic hydatid disease: surgical experience over 15 years. Hepatogastroenterology 2008; 55: 1373-1379.

16. Yorganci K, Sayek I. Surgical treatment of hydatid cysts of the liver in the era of percutaneous treatment. Am J Surg 2002; 184: 63-69.

17. Daradkeh S, El-Muhtaseb H, Farah G, Sroujieh AS, Abu-Khalaf M. Predictors of morbidity and mortality in the surgical management of hydatid cyst of the liver. Langenbecks Arch Surg 2007; 392: 35-39.

18. Dindo D, Demartines N, Clavien PA. Classification of Surgical Complications. A New Proposal With Evaluation in a Cohort of 6336 Patients and Results of a Survey Ann Surg 2004; 240: 205-213.

19. Clavien PA, Barkun J, de Oliveira ML, Vauthey JN, Dindo D, Schulick RD, et al. The Clavien-Dindo classification of surgical complications: five-year experience. Ann Surg 2009; 250: 187-196.

20. Manterola C, Barroso M, Vial M, Bustos L, Muñoz S, Losada $\mathrm{H}$, et al. Liver abscess of hydatid origin: clinical features and results of aggressive treatment. ANZ J Surg 2003; 73: 220-224.

21. Manterola C, Losada H, Carrasco R, Muñoz S, Bustos L, Vial M, y cols. Colangiohidatidosis. Una complicación evolutiva de la hidatidosis hepática. Bol Chil Parasitol 2001; 56: 10-15.

22. Manterola C, Ávila N, Seco J, Ulloa P, Moraga J. Grupo MINCIR. Tránsito hepatotorácico, complicación evolutiva de la hidatidosis hepática. Características clínicas y morbilidad de una serie prospectiva de pacientes intervenidos quirúrgicamente. Rev Chil Cir 2009; 61:
345-349.

23. http://www.cebm.net/index.aspx?o=1025. Accedido el 13 de diciembre de 2009.

24. Manterola C, Vial M, Pineda V, Sanhueza A, Barroso M. Factors associated with morbidity in liver hydatid surgery. ANZ J Surg 2005; 75: 889-892.

25. Manterola C, Bustos L, Vial M, Moraga J. Grupo MINCIR. ¿Es la comunicación quisto-biliar, un factor de riesgo para el desarrollo de morbilidad postoperatoria en pacientes con hidatidosis hepática? Rev Chil Cir 2009; 61: 229-235.

26. Prousalidis J, Kosmidis C, Kapoutzis K, Fachantidis E, Harlaftis N, Aletras H. Intrabiliary rupture of hydatid cysts of the liver. Am J Surg 2009; 197: 193-198.

27. Reza Mousavi S, Khoshnevis J, Kharazm P. Surgical treatment of hydatid cyst of the liver: drainage versus omentoplasty. Ann Hepatol 2005; 4: 272-274.

28. Hofstetter C, Segovia E, Vara-Thorbeck R. Treatment of uncomplicated hydatid cyst of the liver by closed marsupialization and fibrin glue obliteration. World J Surg 2004; 28: 173-178.

29. Franciosi CM, Romano F, Porta G, Caprotti R, De Fina S, Colombo G, et al. Surgical treatment of hydatid disease of the liver. An experience from outside the endemic area. Chir Ital 2002; 54: 667-672.

30. Yüksel O, Akyürek N, Sahin T, Salman B, Azili C, Bostanci H. Efficacy of radical surgery in preventing early local recurrence and cavity-related complications in hydatic liver disease. J Gastrointest Surg 2008; 12 : 483-489.

31. Bektas H, Lehner F, Werner U, Bartels M, Piso P, Tusch $\mathrm{G}$, et al. Surgical therapy of cystic echinococcosis of the liver. Zentralbl Chir 2001; 126: 369-373.

32. Kapan M, Kapan S, Goksoy E, Perek S, Kol E. Postoperative recurrence in hepatic hydatid disease. J Gastrointest Surg 2006; 10: 734-739.

33. Cooney RM, Flanagan KP, Zehyle E. Review of surgical management of cystic hydatid disease in a resource limited setting: Turkana, Kenya. Eur J Gastroenterol Hepatol 2004; 16: 1233-1236. 